differently, but share the unique property that the influence of atomic configuration on physical and mechanical properties can be separated from the influence of variables such as composition, grain size, etc.; and the configuration is changed when an alloy is heat-treated to order it. By quenching out different states of order and testing at ambient temperature, cven the influence of temperature can be separated out.

A substantial body of research on several alloys of the first type had established that the flow stress is a function of degree of order, reaching a maximum at intermediate degrees of order. Numerous interpretations had been advanced to account for this maximum; perhaps the most attractive, Professor Cahn thought, invoked the observed pairing of dislocations to form "super-dislocations" linked by a ribbon of imperfectly ordered alloy. The ribbon broadens as order diminishes and eventually the dislocation pairs disperse; this stage is held to correspond to maximum strength. Other features discussed included the work-hardening capacity of ordered alloys, their strain-ageing behaviour (which revealed a negative correlation between order and rate of recrystallization of a cold-worked alloy), their creep (revealing a pronounced anomaly at the temperature at which order finally disappears) and their fatigue and fracture properties. These last were rationalized in terms of the influence of order on the morphology of plastic slip in alloys. Order totally inhibits eross-slip, which has a crucial influence on both fatigue and fracture.

The second category of alloys-those which change symmetry on ordering - have been much less investigated. Professor Cahn described a recent study by V. S. Arunachalam and himself on the mechanical properties of $\mathrm{CuAu}$, which transforms from cubic (disordered) to tetragonal (ordered). A single cubic grain acquires a system of microstrains because of the shape changes associated with ordering in the various microdomains into which the crystal decomposes. These microstrains produce hardening; if they become too large, spontaneous fracture may also ensue. Strainageing behaviour is quite different from the first category of alloys. A strange consequence of the change of shape of the unit cell is that a very small elastic stress applied during ordering can completely change the morphology of ordered domains and alter both the dimensions of the entire sample and also its mechanical properties. In Professor Cahn's view, this novel form of 'superplasticity', and indeed the behaviour of this class of alloys generally, deserves more attention than it has had in the past.

\section{Bacteria and Plastics}

\section{from a Correspondent}

The Microbiology Group of the Society of Chemical Industry held a meeting on Thursday, November 2, on the Biodeterioration of Rubbers and Plastics. First Dr G. H. Booth and Mr J. A. Robb of the National Physical Laboratory read a joint paper on the bacterial degradation of plasticized PVC, describing their experiments with five $\mathrm{PVC}$ polymers, 13 plasticizers and two stabilizers. Films were buried at $22^{\circ}-25^{\circ} \mathrm{C}$ in neutral soil which had been enriched with bacterial cultures from a variety of sources. When diiso-octyl phthalate was used as the plasticizer and white lead as stabilizer, the change in properties after burial was relatively small for all fine polymers. One of the homopolymers was then combined with each plasticizer in turn, using white lead as a stabilizer, and finally the series was repeated with a cadmium stabilizer. When di-ammonium phosphate or a chlorinated hydrocarbon was used as plasticizer, the change in properties was less for the cadmium-stabilized film than for the leadstabilized film, suggesting that the cadmium compound was more toxic than the lead compound towards the bacteria. The fact that the degree of attack depended on the plasticizer suggested that it was this which was being consumed in all cases.

Dr S. H. Morrell of the Rubber and Plastics Research Association, Shawbury, Shropshire, gave a general paper reviewing the effects of micro-organisms, principally fungi, on rubbers and plastics. He reminded his audience that rubbers and plastics were often complex materials containing inorganic as well as organic additives, and suggested that reported attacks might well be confined to consumption of these additives rather than destruction of the polymer. In some cases the attack had been exaggerated, incidents of "failure" being quoted when the material was well able to give good service for some considerable time.

There was a detailed description of the various rubbers and plastics, together with comments on the chemical nature of the material and possible reasons for its attack or otherwise by micro-organisms. It was concluded that evidence of attack was sometimes confused and contradictory, and in some cases it was necessary to distinguish between actual attack and growth on the surface of the material. It could not be said that only naturally occurring polymers were attacked, nor was it possible at this stage to say which chemical groups in each polymer were susceptible. The meeting concluded with a vigorous discussion on both papers.

\section{Simulated Growth and Morphogenesis}

\section{from our Microbiology Correspondent}

THE behaviour of complex systems, most prominently illustrated by those of air and road traffic control, can be analysed effectively by simulation techniques. The usefulness of such studies is determined by the number of parameters of the system which can be programmed. In biological systems the parameters of growth and morphogenesis are inadequately defined and complete simulations are not feasible. Biological patterns can, however, be studied with computers and two recent papers are sound advocates of this approach.

In this journal Cohen has reported attempts to simulate two-dimensional branching patterns of the type produced by surface cultures of fungi and the vascular systems in leaves (Nature, 216, 246; 1967). Programmes were written for each class of pattern to be simulated, the rules being determined from acceptable hypotheses of the natural system concerned. Cohen limited his analyses to patterns based upon apical growth and sub-apical branching and examined the introduction of four types of effect: (1) varied growth parameters in branches of different rank, an effect producing hierarchical organization; (2) changing parameters during the course of growth; (3) influence of branch length on the extent of growth and branching; and (4) local disturbances in the growth domain. The 